\title{
Meta-analysis identifies candidate key genes in endometrium as predictive biomarkers for clinical pregnancy in IVF
}

\author{
Jingyu Li ${ }^{1}$, Dongyun Liu ${ }^{1}$, Jiang Wang ${ }^{1}$, Huali Deng ${ }^{1}$, Xiu Luo ${ }^{1}$, Xiaoli Shen ${ }^{1}$, Yanjun \\ Huan ${ }^{2}$, Guoning Huang ${ }^{1}$ and Hong $\mathbf{Y e}^{1}$ \\ ${ }^{1}$ Chongqing Reproductive and Genetics Institute, Yu Zhong District, Chongqing, China \\ ${ }^{2}$ College of Veterinary Medicine, Qingdao Agricultural University, Qingdao, China \\ Correspondence to: Guoning Huang, email: gnhuang217@sina.com \\ Hong Ye, email: yehong1210@163.com
}

Keywords: implantation failure, endometrial receptivity, microarray, meta-analysis, IVF

Received: August 09, 2017

Accepted: September 22, 2017

Published: October 26, 2017

Copyright: Li et al. This is an open-access article distributed under the terms of the Creative Commons Attribution License 3.0 (CC BY 3.0 ), which permits unrestricted use, distribution, and reproduction in any medium, provided the original author and source are credited.

\section{ABSTRACT}

Genetic factors in endometrium are likely to be involved in the embryo implantation failure (IF), one of the major limiting factors in the success of in vitro fertilization (IVF). In this study, we aimed to identify critical genes from the transcriptional profile for the establishment of the endometrial receptivity which supporting the normal pregnancy. Three GEO datasets, including 12 samples of IF and 12 samples of controls, were used for the meta-analysis. We identified 182 different expression genes (DEGs) by comparing IF with controls and present here the successful clustering according to sample type, not by the origin. The gene ontology (GO) enriched analysis demonstrated the significant downregulation in activation and regulation of inflammatory and immune response in IF patients. Furthermore, network analysis of down-regulated genes identified the significant hub genes containing GADD45A (growth arrest and DNA damage inducible alpha, Degree $=77$ ), GZMB (granzyme B, Degree $=38$ ) and NLRP2 (NLR family pyrin domain containing 2 , Degree = 37). The lower expression of NLRP2, related to inflammatory responses with the most degree in the network, was validatied by other GEO data. Besides, it was confirmed that the NLRP2 could act as a predictor for pregnancy after IVF (AUC = $87.93 \%$; sensitivity, $60.00 \%$; specificity, $91.30 \%$ ). Our meta-analysis will help us to better understand the molecular regulation of endometrial receptivity, and guiding further line of treatment for IF during IVF.

\section{INTRODUCTION}

Despite of advancement in the assisted reproductive techniques (ART), current pregnancy and live birth success rates still remain unsatisfactory $[1,2]$. The major reason for this limited success is embryo implantation failure (IF), which is mainly caused by the low-quality embryo and impaired endometrial receptivity [3]. The embryo quality has been the most focused player in this process, with many decades of embryological research [4-7]. However, a significant proportion of couples still undergoing IVF experience IF, even after the good-quality embryo transfer. Endometrial dysfunction could be one of the major factors related to the IF in this condition [3].
Therefore, elucidation of the molecular mechanisms controlling endometrial receptivity is important for improving the success rate of ART.

Embryo implantation can only occur during a temporally restricted period from day 20 to day 24 of the menstrual cycle, called the window of implantation (WOI) [8]. The endometrium is nonreceptive in the most of the menstrual cycle, prohibiting the adherence and implantation proceeding. During the WOI, however, the endometrium is receptive and permits a blastocyst to attach and invade. Previous studies have indicated the important roles of inflammatory and immune response in embryo implantation, through allowing the invasion and maintenance of semi-allogenic embryo in the endometrium 
$[9,10]$. In addition, several biological processes including cell proliferation $[11,12]$, cytokines and estradiol response have been considered to be responsible for successful implantation [11, 13, 14]. Thus, the precise regulation of gene expression during WOI is required for the establishment of endometrial receptivity.

High-throughput technology is useful for monitoring the transcriptomes of endometrium during the WOI. Recent studies have enabled advances in understanding the molecular mechanisms of endometrial receptivity and demonstrated the feasibility of the diagnosis of endometrial receptivity status with the use of transcriptomic profiling [12, 15-19]. Although many long lists of key gene signatures of endometrial receptivity were identified, there tends to be inconsistencies among studies due to the differences in experimental designs, microarray type, day of the cycle when the sample is collected, and other reasons [12, 15-19]. To address these challenges, meta-analysis has been applied for large-scale comparative analysis of multiple gene expression datasets, which can enhance statistical power in identifying more robust and reliable gene signatures [20]. However, similar meta-analysis has never been conducted for IF.

In this study, we performed the first meta-analysis of endometrial gene expression datasets from various IF studies to overcome the limitation of heterogeneity. We focused our main attention on genes that are downregulated, since a strong skew towards down-regulation of processes in IF patients was detected through Gene Ontology (GO) enrichment analysis. Network analysis identified NLPR2 (NLR family pyrin domain containing 2 ), as the most important hub gene related to inflammatory response. Moreover, receiver operating characteristic (ROC) analysis of NLRP2 suggested its potential to be a single predictive biomaker for clinical pregnancy in IVF. These findings deepened the understanding of the molecular mechanisms of endometrial receptivity, and identifying a novel list of candidate key genes associated with IF.

\section{RESULTS}

\section{Studies included in the meta-analysis}

A total of 116 datasets were identified by electronic search, 108 of which were excluded because 53 were animal studies, 13 were not mRNA expression array, and 42 didn't contained endometrial samples. Thus, 8 microarray datasets were selected for full-text data review for more detailed evaluation. Five datasets were excluded because 1 microarray arary platform didn't meet the requirement, and the endometrial samples in the other 4 microarray datasets were not from IF and control patients in IVF cycle. Finally, three microarray datasets met the inclusion criteria and were considered for subsequent analysis. The flowchart outlining the selection process in detail is shown in Figure 1. The details of the individual microarray dataset analyzed in this study are summarized in Table 1.

\section{Meta-analysis of endometrial gene expression patterns in control vs. IF patient}

Meta-analysis using a rank product method identified a total of 182 genes consistently differentially expressed in IF group compared with controls across three microarray datasets (pfp $<0.05)$. Among the 182 candidate implantation-associated genes, 119 genes were up-regulated and 63 were down-regulated in IF patients compared with controls. The complete list of DEs is provided in Supplementary Table 1. The downregulated gene with the lowest pfp (average $\mathrm{FC}=10.13$, pfp $=3.97 \times 10^{-13}$ ) was PAEP (progestagen associated endometrial protein), which is known to play an important role in regulating the endometrial environment suitable for implantation via immunomodulatory mechanisms [21]. A heat map visualization of the DEs across the three datasets is displayed in Figure 2A. To ascertain whether the expression profiles of DEs can distinguish the IF from control patient, we performed the unsupervised hierarchical clustering. Overall, the same sample type clustered together, with the exception that three IF samples clustered in control group and one control sample in IF group (Figure 2B).

\section{Functional analysis}

To identify biological processes associated with gene expression differences in control vs. IF patient, we performed GO enrichment analysis with the up- and down-regulated genes separately. Interestingly, there were many more enriched GO terms with down-regulated genes (66\%, Figure 3), indicating that the down-regulation of genes in endometrium might be the underlying main cause for implantation failure. Therefore we focused on the down-regulated genes and found that GO terms for biological process significantly enriched in inflammatory (GO:0006954, $P=1.91 \times 10^{-3}$ ) and immune response (GO: $0006955, P=1.49 \times 10^{-2}$ ), while for molecular functions, the enriched GO terms were heparin binding (GO:0004364, $P=1.43 \times 10^{-4}$ ) and calcium ion binding (GO:0005509, $\left.P=3.54 \times 10^{-4}\right)$, and for cellular component, the enriched GO terms were extracellular region (GO:0005576, $P=4.67 \times 10^{-10}$ ) and golgi lumen (GO:0005796, $\left.P=2.81 \times 10^{-4}\right)$. The processes of embryo implantation are considered analogous to inflammatory and immune responses $[9,16,22,23]$, thus we speculated that these down-regulation genes involved in inflammatory and immune responses might play important roles in regulating the endometrial receptivity for implantation. 
Table 1: Microarray studies in endometrium used for analysis

\begin{tabular}{lll}
\hline GEO accession no. & No. of samples & Platform \\
\hline GSE18140 & Pregnant $=4$; Nonpregnant $=4$ & GPL570 [HG-U133_Plus_2] Affymetrix \\
& & Human Genome U133 Plus 2.0 Array \\
GSE21225 & Pregnant $=3$; Nonpregnant $=3$ & GPL570 [HG-U133_Plus_2] Affymetrix \\
& & Human Genome U133 Plus 2.0 Array \\
GSE26787 & Pregnant $=5$; Nonpregnant $=5$ & GPL570 [HG-U133_Plus_2] Affymetrix \\
& & Human Genome U133 Plus 2.0 Array \\
\hline
\end{tabular}

\section{Network analysis}

To further predict the function of these downregulated genes in IF patients, a network analysis was performed. The resulting network contained 434 nodes and 484 edges (Figure 4A). The most highly ranked genes containing GADD45A (growth arrest and DNA damage inducible alpha, Degree $=77), G Z M B$ (granzyme $\mathrm{B}$, Degree $=38$ ) and NLRP2 (NLR family pyrin domain containing 2, Degree $=37$ ) (Supplementary Table 2). To investigate the functions of the 434 genes in the network, we mapped them to the Kyoto Encyclopedia of Genes (KEGG) database. Hypergeometric test with $P$ value $<0.05$ was used as the criteria for pathway detection (Supplementary Table 3 ). The results showed that majority of the enrichments were inflammation and immune related pathways, for instance, the $\mathrm{T}$ cell receptor signaling pathway (Figure 4B). In addition, we also identified cell cycle pathyway, indicative of a reduced rate of cellular proliferation in endometrium. This is consistent with previous studies of IF that also reported cell cycle enrichment for the down-regulated genes [12].

\section{Validation of the expression of $N L R P 2$}

The previous reports have suggested that controlled inflammation and activation of the immune response is essential for embryo implantation. Thus, we selected $N L P R 2$, involved in inflammatory responses with the most degree in the network, for the validation of different expression. Here, we performed the expression quantification analysis for NLPR2 in endometrium between IF and control patients using another nonGPL570 microarray data. The expression results revealed that $N L P R 2$ was significantly down-regulated in IF patients (Figure 5A). Furthermore, ROC analysis of

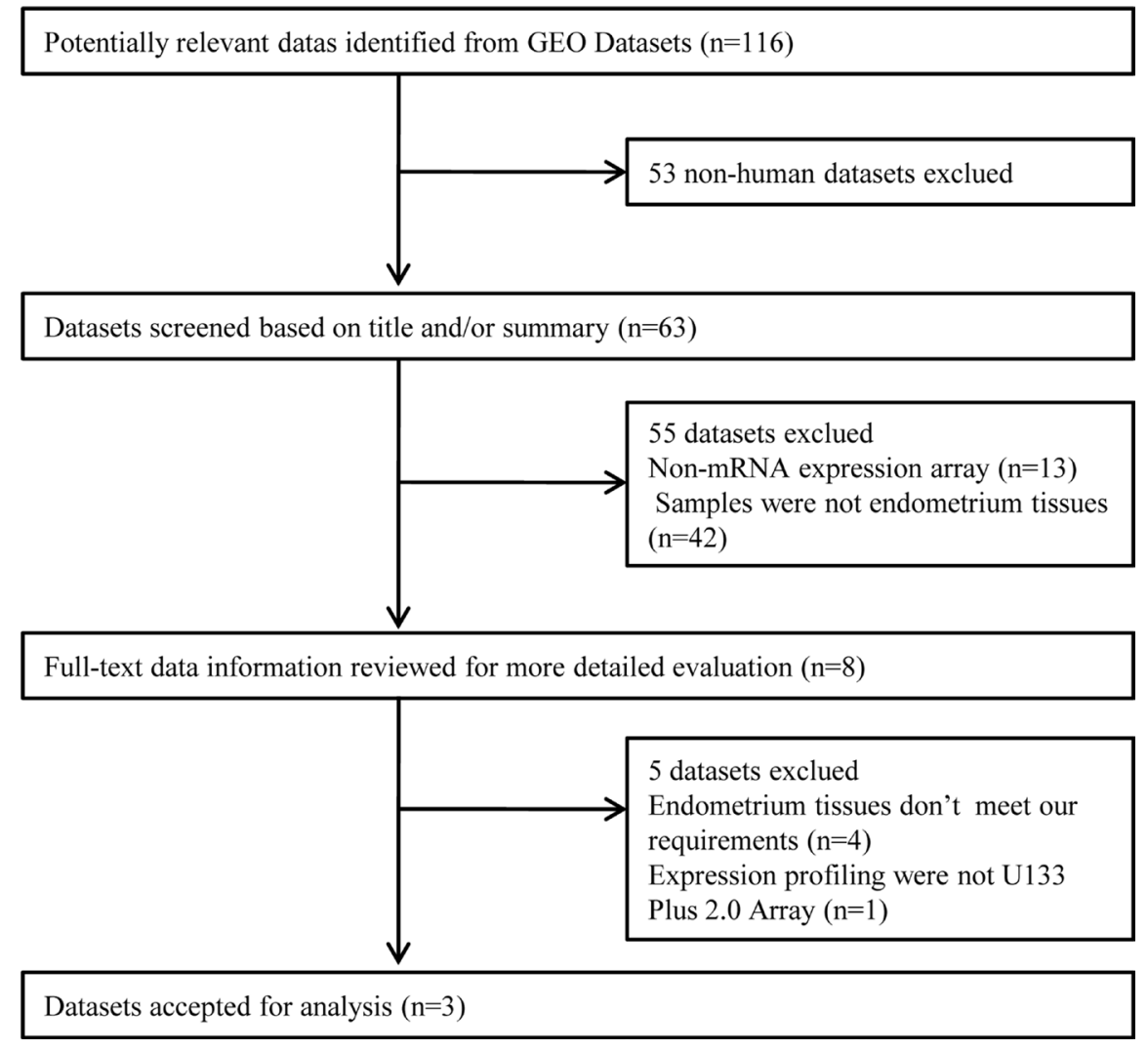

Figure 1: Flowchart of the selected process of microarray datasets for the meta-analysis. 
$N L R P 2$ was performed to evaluate the diagnostic accuracy. The area under the curve was $87.93 \%$, and the sensitivity and specificity reached $60.0 \%$ and $91.3 \%$ (Figure 5B).

\section{DISCUSSION}

Many genes have been identified as molecular biomarkers of endometrial receptivity using highthroughput technology [12, 15-19]. However, the large number of biomarkers and the lack of explanation about their interactions make it extremely difficult to display a comprehensive overview of the process in a holistic way. Therefore, it is extremely important to identify the consistent biomarkers of endometrial receptivity. A meta-analysis that includes massive amounts of data from publicly available transcriptomes will be more accurate and improve biological understanding compared with individual analysis $[20,24]$. Here, we employed a meta-analysis strategy on combined DEGs that were collected from different origins to highlight genes that were consistently differentially expressed with statistical significance between IF and control patients.

An appropriate analysis strategy and statistical methodology is crucial for the meta-analysis. Here, we normalized these expression datasets separately for addressing heterogeneity. In this condition, any measurement errors from a single data would have no influence on the final results with our normalization strategy [25]. For statistical analysis, we applied the nonparametric rank product method because it outperforms the other methods in terms of sensitivity and specificity, especially among multiple datasets [20, 26, 27]. Overall, our analysis strategy properly reduced the effect of heterogeneity derived from the different datasets and provided control over the extent to which false positives were included.

In total, we identified 182 genes that were differentially expressed in IF patients compared to control, including 119 up-regulated and 63 down-regulated genes. Using the expression patterns of these DEGs,

\section{A}

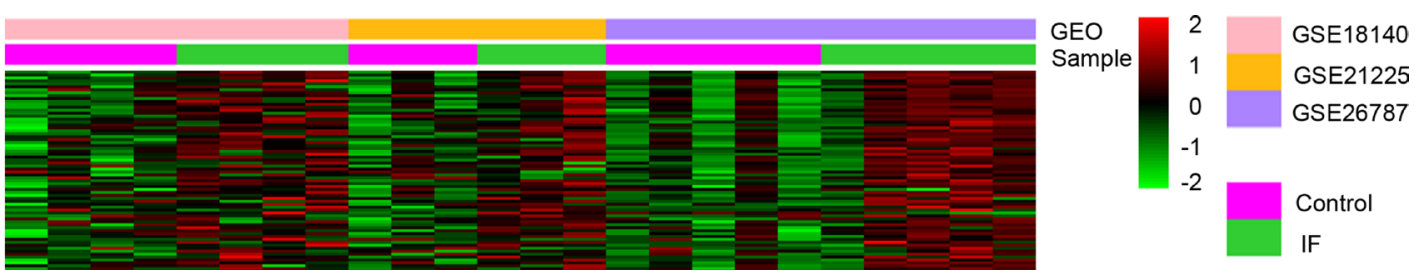

B

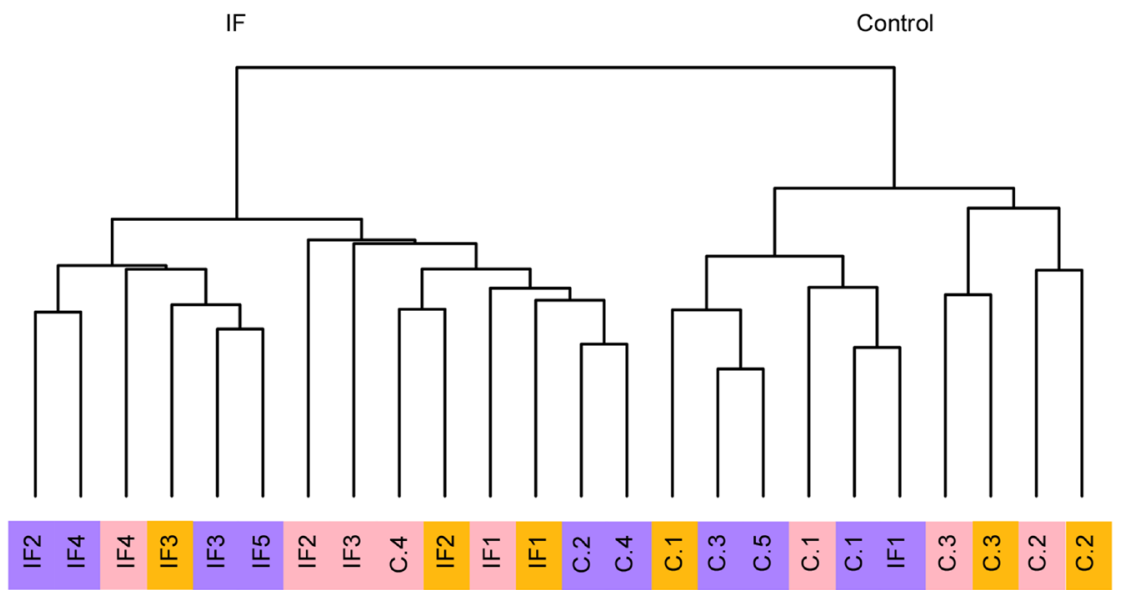

GSE18140

GSE21225

GSE26787

Figure 2: Genes differentially expressed in endometrium between IF and control patients across three datasets. (A) Heat map representation of the DEGs between control and IF patients across different microarrays identified from the meta-analysis. Each color above represents a single dataset. The heat map was rescaled to prevent domination by study-specific effects. (B) Unsupervised clustering of the transcriptome of the DEGs in the three datasets. 
we successfully distinguished the IF from the control patients through unsupervised hierarchical clustering, suggesting the potential of the DEGs to be the biomarkers of endometrial receptivity. An important gene PAEP was identified as the most down-regulated in our meta-analysis. Progesterone secreted by corpus luteum stimulates the expression of PAEP in WOI . It is also reported to have high expression in endometrium and was down regulated in IF patients undergoing IVF cycle [28-31]. Therefore, the down-regulation of $P A E P$ leads to the endometrial deregulation in IF patients.

GO enrichment analysis displayed a strong skew towards down-regulation of processes in IF patients. This is consistent with previous studies of IF that reported enrichment for inflammatory and immune response within the set of down-regulated genes [16]. In addition to this, the KEGG pathyway analysis of network also showed the dysregulated pathyways, including $\mathrm{T}$ cell and B cell receptor signaling pathway (Figure 4B), Natural killer cell pathway and myeloid leukemia, indicating the downregulated function of positive regulation of inflammatory and immune response during WOI. Rencent studies have demonstrated that the down-regulation of inflammation might lead to the impaired elicitation of immune cells as well as their recruitment to the endometrium [32, 33]. Thus, this would be the main cause of defect in creating suitable environment in endometrium during
WOI leading to IF. Furthermore, we detected the downregulation in IF patients of genes involved in cell cycle and apoptosis regulation (Supplementary Table 3), indicative of a reduced rate of cell proliferation in endometrium. Additionally, in this meta-analysis, the down-regulation genes in IF patients were also enriched in estradiol response, chemokines, growth factors, which have been found to be important for the maintenance of microenvironment during implantation supporting the endometrium-embryo crosstalk [34, 35].

Based on network analysis, NLPR2 was identified as the most significant hub gene in inflammatory pathway. NLRP family are important regulatory factors of innate immunity and inflammation [36, 37]. Interestingly, maternal deficiency of NLRP2 can cause embryonic lethality in mice $[38,39]$, suggesting its vital roles in reproduction. However, till data there is no reports about the association between NLRP2 and endometrial receptivity. A biomarker-based diagnose for endometrial receptivity could have great impact in clinical scenario. Here, the evaluation of biomarker performance showed that $N L R P 2$ can distinguish IF patients from controls with $60.0 \%$ sensitivity and $91.3 \%$ specificity. However, validation of the biomarker in prospective cohorts of IVF patients would be required.

In summary, we performed a meta-analysis of endometrial gene expression and identified a list of

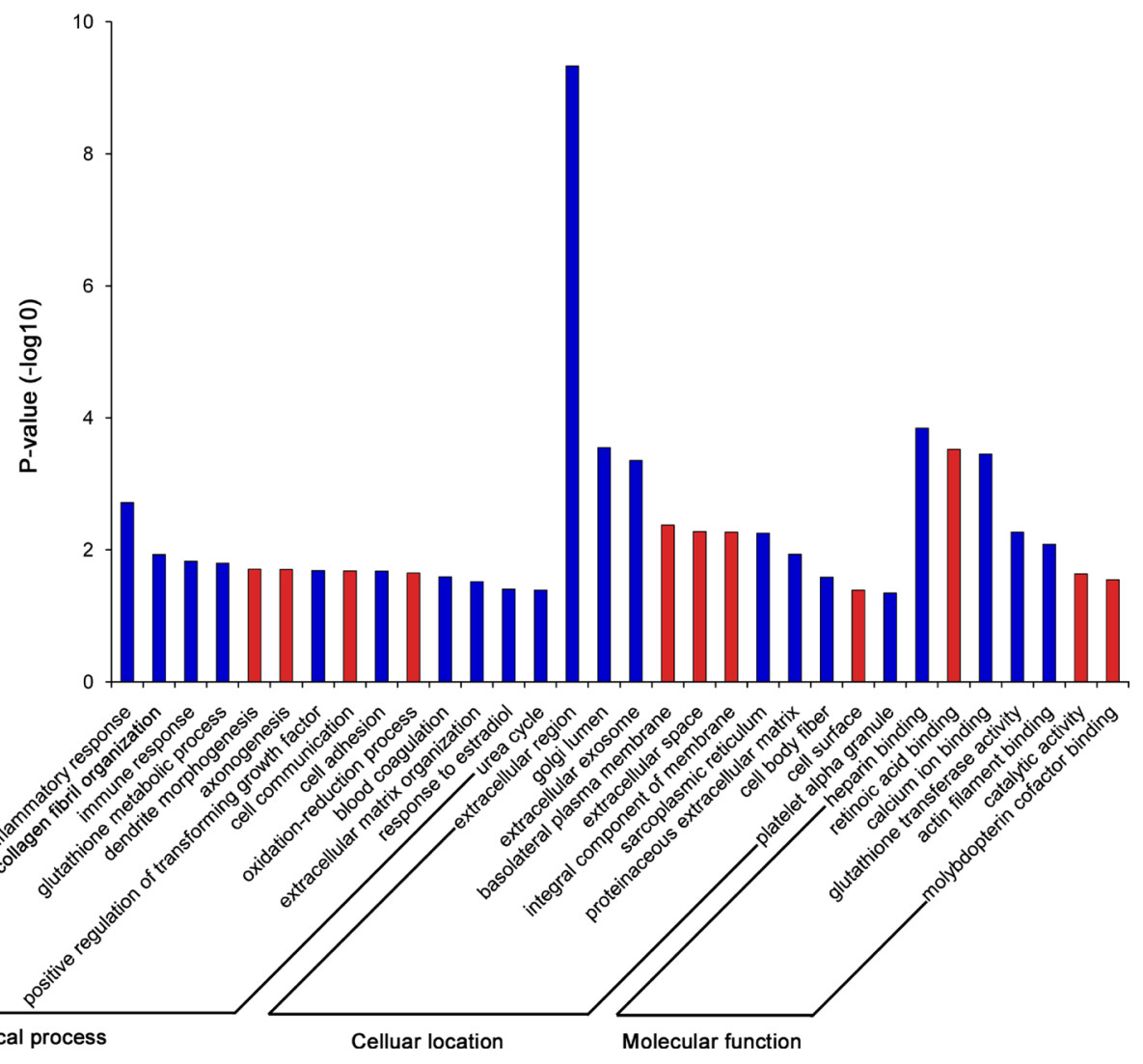

Figure 3: GO enrichment analysis for the up- and down-regulated genes. 
important gene signature that might contribute to the endometrial deregulation in IF patients. In addition, our results strengthen the association between endometrial receptivity and several pathyways, especially the inflammatory and immune pathways, and provide insights into the molecular mechanisms underlying the impairment of inflammatory and immune signaling observed in IF patients. Furthermore, this study underscores the potential of network analysis as a powerful framework to gain insight into the mechanisms underlying endometrial receptivity and to identify potential biomarkers. Further functional studies and validated test about the role of these biomarkers in endometrial receptivity are warranted.

\section{MATERIALS AND METHODS}

\section{Identification of eligible gene expression dataset}

To compare the difference of human endometrium derived from control and IF patients in IVF cycle, the NCBI GEO (Gene Expression Omnibus) database (http:// www.ncbi.nlm.nih.gov/geo/) was searched for eligible expression datasets using the key words 'endometrium' and 'microarray' on June 1, 2017. Datasets that met the following inclusion criteria were included: 1) all two types of endometrium tissue (IF and control) were contained in one experiment; 2) GeneChip Human Genome U133
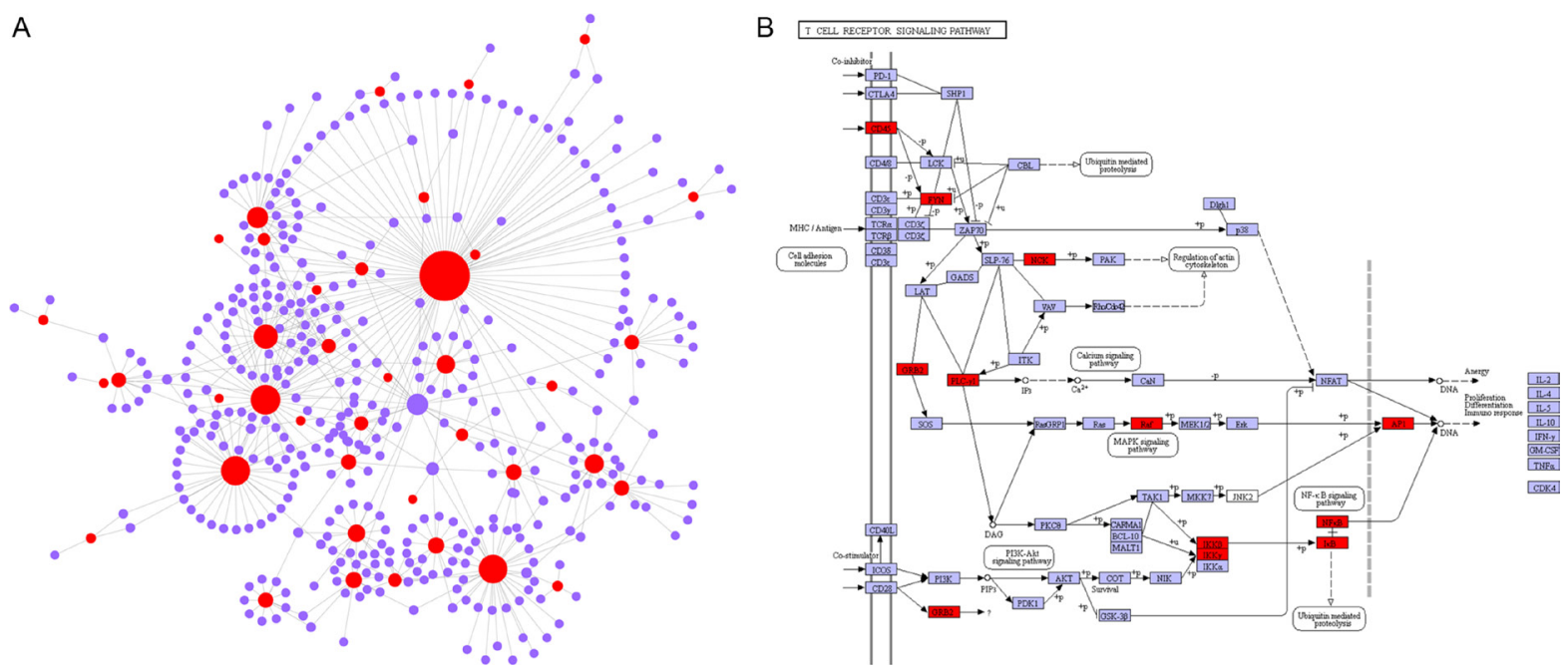

Figure 4: The network analysis of down-regulated genes in endometrium of IF patients. (A) Network including 434 nodes and 484 edges. Red: down-regulated genes. Purple: interaction genes. (B) The network genes are enriched in T cell receptor signaling pathway. Red rectangles represent the genes in the network. Purple is the color of Kyoto Encyclopedia of Genes and Genomes (KEGG) database.
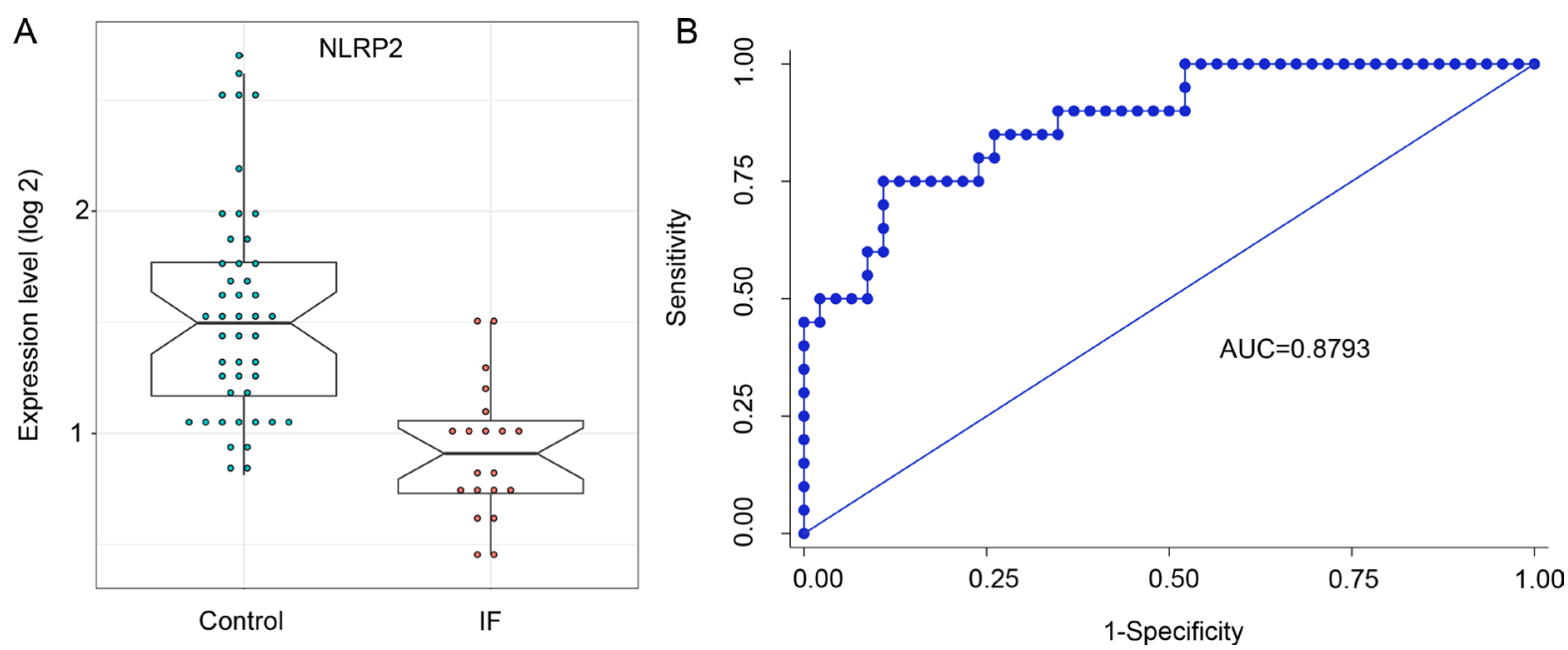

Figure 5: Evaluation of NLRP2 as predictive biomarker for clinical pregnancy in IVF. (A) Relative abundance of $N L R P 2$ in endometrium of IF patients (red circles) compared with control (green circles) in samples from the datasets of GSE58144. A Student $\mathrm{T}$ test (two-tailed) was used to estimate the significance between IF and control patients. (B) ROC curves analysis for clinical pregnancy prediction by NLRP2 expression in endometrium. 
Plus 2.0 arrays GPL570 platform; 3) there were at least three replicates in each sample. Data were extracted from the original studies by two independent reviewers. Any discrepancies between reviewers were resolved by consensus or a third reviewer. The microarray information of the GPL15789 microarray platform for results validation in this study was obtained from GSE58144.

\section{Meta-analysis of microarray datasets}

A total of three microarray datasets passed the inclusion criteria and were considered for subsequent analysis. The datasets analyzed in this study are listed in Table 1. For the microarray analyses, the raw data were $\log 2$-transformed and quantile normalized independently for individual datasets using RMA in R (Bioconductor). When multiple probes were mapped to the same gene, those probes were averaged. To avoid bias from the heterogeneity among multiple datasets, we used a nonparametric approach based on rank order in the RankProd package to detect differentially expressed genes (DEs) that were consistently highly ranked in different datasets. Briefly, this method combines the gene rank from different datasets together to select the DE. The fold changes (FCs) are computed for all possible pairwise comparisons. The ranks of the FCs within each comparison are then used to calculate the rank product foreach gene. We used 1000 permutations to obtain the percentage of false positive predictions (pfp), which is also known as the false discovery rate (FDR). As recommended, a pfp value of 0.05 was used to set the threshold for DEs.

\section{Function enrichment analysis}

The Database for Annotation, Visualization and Integrated Discovery (DAVID) was a frequently-used bioinformatics resources for GO functional annotation. First, we upload gene lists to DAVID. And then, after selecting identifier for thes genes (In this work, we select "OFFICIAL_GENE_SYMBOL). Biological process, molecular fuction and cellular component terms was seleted as background gene sets respectively. Hypergeometric Exact test was used to measure geneenrichment in background annotation terms.

\section{Network analysis}

We used a comprehensive high-quality PPI database downloaded from the InnateDB [30], which participates in the International Molecular Exchange (IMEx) consortium [31]. The database was derived by manually curating protein interaction data from published literature and by integrating experimental data from several PPI databases including MINT [32], IntAct [33], BIND, BioGRID [34] and DIP [35]. The gene networks were visualized using Cytoscape 3.2.0 [36].

\section{Statistical analysis}

Statistical analysis was performed using STATA12.0 software (Statacorp, TX, USA). The Student $t$ test (twotailed) was used to estimate the significance between IF and control patients for numerical variables. Receiver operating characteristic curves (ROC) were used to evaluate the prognostic ability of biomarker to successful pregnancy. The area under the ROC curve and the sensitivity and specificity were also calculated.

\section{Abbreviations}

IF: implantation failure; ART: assisted reproductive techniques; IVF: in vitro fertilization (IVF); DEGs: differentially expressed genes.

\section{Author contributions}

H.Y., G.N.H. and J.Y.L. supervised this work. J.Y.L. designed the research and performed data collection and data analysis. J.W. helped with the data analysis. J.Y.L. prepared figures and writed the manuscript. D.Y.L, X.L.S and X.L. polished the manuscript. J.Y.L., G.N.H. and H.Y reviewing the manuscript. All authors contributed to the manuscript at various stages.

\section{CONFLICTS OF INTEREST}

The authors declare no competing financial interests.

\section{FUNDING}

This work was supported by grants from Chongqing Municipal Health and Family Planning Commission (2012-1-073, 2013-2-130, 2015MSXM085), Chongqing YuZhong Science Project (20170127), and Chongqing social undertakings and people's livelihood guarantee scientific innovation technology (cstc2015shms-ztzx0031).

\section{REFERENCES}

1. Mascarenhas MN, Flaxman SR, Boerma T, Vanderpoel $\mathrm{S}$, Stevens GA. National, regional, and global trends in infertility prevalence since 1990: a systematic analysis of 277 health surveys. PLoS Med. 2012; 9:e1001356.

2. Smith JF, Eisenberg ML, Millstein SG, Nachtigall RD, Sadetsky N, Cedars MI, Katz PP. Fertility treatments and outcomes among couples seeking fertility care: data from a prospective fertility cohort in the United States. Fertil Steril. 2011; 95:79-84.

3. Achache H, Revel A. Endometrial receptivity markers, the journey to successful embryo implantation. Hum Reprod Update. 2006; 12:731-46. 
4. Montag M, Toth B, Strowitzki T. New approaches to embryo selection. Reprod Biomed Online. 2013; 27:539-46.

5. Armstrong S, Arroll N, Cree LM, Jordan V, Farquhar C. Time-lapse systems for embryo incubation and assessment in assisted reproduction. Cochrane Database Syst Rev. 2015: CD011320.

6. Kaser DJ, Racowsky C. Clinical outcomes following selection of human preimplantation embryos with timelapse monitoring: a systematic review. Hum Reprod Update. 2014; 20:617-31.

7. Wong CC, Loewke KE, Bossert NL, Behr B, De Jonge CJ, Baer TM, Reijo Pera RA. Non-invasive imaging of human embryos before embryonic genome activation predicts development to the blastocyst stage. Nat Biotechnol. 2010; 28:1115-21.

8. Wilcox AJ, Baird DD, Weinberg CR. Time of implantation of the conceptus and loss of pregnancy. N Engl J Med. 1999; 340:1796-9.

9. Robertson SA, Moldenhauer LM. Immunological determinants of implantation success. Int J Dev Biol. 2014; 58:205-17.

10. van Mourik MS, Macklon NS, Heijnen CJ. Embryonic implantation: cytokines, adhesion molecules, and immune cells in establishing an implantation environment. J Leukoc Biol. 2009; 85:4-19.

11. Koler M, Achache H, Tsafrir A, Smith Y, Revel A, Reich R. Disrupted gene pattern in patients with repeated in vitro fertilization (IVF) failure. Hum Reprod. 2009; 24:2541-8.

12. Koot YE, van Hooff SR, Boomsma CM, van Leenen D, Groot Koerkamp MJ, Goddijn M, Eijkemans MJ, Fauser BC, Holstege FC, Macklon NS. An endometrial gene expression signature accurately predicts recurrent implantation failure after IVF. Sci Rep. 2016; 6:19411.

13. Lee JH, Kim TH, Oh SJ, Yoo JY, Akira S, Ku BJ, Lydon JP, Jeong JW. Signal transducer and activator of transcription-3 (Stat3) plays a critical role in implantation via progesterone receptor in uterus. FASEB J. 2013; 27:2553-63.

14. Pawar S, Starosvetsky E, Orvis GD, Behringer RR, Bagchi IC, Bagchi MK. STAT3 regulates uterine epithelial remodeling and epithelial-stromal crosstalk during implantation. Mol Endocrinol. 2013; 27:1996-2012.

15. Lédée N, Munaut C, Aubert J, Sérazin V, Rahmati M, Chaouat G, Sandra O, Foidart JM. Specific and extensive endometrial deregulation is present before conception in IVF/ICSI repeated implantation failures (IF) or recurrent miscarriages. J Pathol. 2011; 225:554-64.

16. Pathare ADS, Zaveri K, Hinduja I. Downregulation of genes related to immune and inflammatory response in IVF implantation failure cases under controlled ovarian stimulation. Am J Reprod Immunol. 2017; 78.

17. Diaz-Gimeno P, Ruiz-Alonso M, Blesa D, Bosch N, Martinez-Conejero JA, Alama P, Garrido N, Pellicer A, Simon C. The accuracy and reproducibility of the endometrial receptivity array is superior to histology as a diagnostic method for endometrial receptivity. Fertil Steril. 2013; 99:508-17.

18. Ruiz-Alonso M, Blesa D, Diaz-Gimeno P, Gomez E, Fernandez-Sanchez M, Carranza F, Carrera J, Vilella F, Pellicer A, Simon C. The endometrial receptivity array for diagnosis and personalized embryo transfer as a treatment for patients with repeated implantation failure. Fertil Steril. 2013; 100:818-24.

19. Van Vaerenbergh I, Blockeel C, Van Lommel L, Ghislain V, In't Veld P, Schuit F, Fatemi HM, Devroey P, Bourgain C. Cyclooxygenase-2 network as predictive molecular marker for clinical pregnancy in in vitro fertilization. Fertil Steril. 2011; 95:448-51, 51 e1-2.

20. Hong F, Breitling R. A comparison of meta-analysis methods for detecting differentially expressed genes in microarray experiments. Bioinformatics. 2008; 24:374-82.

21. Seppala M, Koistinen H, Koistinen R, Hautala L, Chiu PC, Yeung WS. Glycodelin in reproductive endocrinology and hormone-related cancer. Eur J Endocrinol. 2009; 160:121-33.

22. Kimber SJ. Leukaemia inhibitory factor in implantation and uterine biology. Reproduction. 2005; 130:131-45.

23. Giuliani E, Parkin KL, Lessey BA, Young SL, Fazleabas AT. Characterization of uterine NK cells in women with infertility or recurrent pregnancy loss and associated endometriosis. Am J Reprod Immunol. 2014; 72:262-9.

24. Choi JK, Yu U, Kim S, Yoo OJ. Combining multiple microarray studies and modeling interstudy variation. Bioinformatics. 2003; 19:i84-90.

25. Gao F, Li J, Zhang H, Yang X, An T. Identifying Candidate Reprogramming Genes in Mouse Induced Pluripotent Stem Cells. Stem Cell Rev. 2017; 13:532-541.

26. Breitling R, Armengaud P, Amtmann A, Herzyk P. Rank products: a simple, yet powerful, new method to detect differentially regulated genes in replicated microarray experiments. FEBS Lett. 2004; 573:83-92.

27. Hong F, Breitling R, McEntee CW, Wittner BS, Nemhauser JL, Chory J. RankProd: a bioconductor package for detecting differentially expressed genes in meta-analysis. Bioinformatics. 2006; 22:2825-7.

28. Liu Y, Lee KF, Ng EH, Yeung WS, Ho PC. Gene expression profiling of human peri-implantation endometria between natural and stimulated cycles. Fertil Steril. 2008; 90:2152-64.

29. Bhagwat SR, Chandrashekar DS, Kakar R, Davuluri S, Bajpai AK, Nayak S, Bhutada S, Acharya K, Sachdeva G. Endometrial receptivity: a revisit to functional genomics studies on human endometrium and creation of HGExERdb. PLoS One. 2013; 8:e58419.

30. Talbi S, Hamilton AE, Vo KC, Tulac S, Overgaard MT, Dosiou C, Le Shay N, Nezhat CN, Kempson R, Lessey BA, Nayak NR, Giudice LC. Molecular phenotyping of human endometrium distinguishes menstrual cycle phases and underlying biological processes in normo-ovulatory women. Endocrinology. 2006; 147:1097-121. 
31. Altmae S, Reimand J, Hovatta O, Zhang P, Kere J, Laisk T, Saare M, Peters M, Vilo J, Stavreus-Evers A, Salumets A. Research resource: interactome of human embryo implantation: identification of gene expression pathways, regulation, and integrated regulatory networks. Mol Endocrinol. 2012; 26:203-17.

32. Saito S, Nakashima A, Shima T, Ito M. Th1/Th2/Th17 and regulatory T-cell paradigm in pregnancy. Am J Reprod Immunol. 2010; 63:601-10.

33. Blois SM, Kammerer U, Alba Soto C, Tometten MC, Shaikly V, Barrientos G, Jurd R, Rukavina D, Thomson AW, Klapp BF, Fernandez N, Arck PC. Dendritic cells: key to fetal tolerance? Biol Reprod. 2007; 77:590-8.

34. Salamonsen LA, Evans J, Nguyen HP, Edgell TA. The Microenvironment of Human Implantation: Determinant of Reproductive Success. Am J Reprod Immunol. 2016; 75:218-25.
35. Bhusane K, Bhutada S, Chaudhari U, Savardekar L, Katkam R, Sachdeva G. Secrets of Endometrial Receptivity: Some Are Hidden in Uterine Secretome. Am J Reprod Immunol. 2016; 75:226-36.

36. Kufer TA, Sansonetti PJ. NLR functions beyond pathogen recognition. Nat Immunol. 2011; 12:121-8.

37. Strowig T, Henao-Mejia J, Elinav E, Flavell R. Inflammasomes in health and disease. Nature. 2012; 481:278-86.

38. Peng H, Chang B, Lu C, Su J, Wu Y, Lv P, Wang Y, Liu J, Zhang B, Quan F, Guo Z, Zhang Y. Nlrp2, a maternal effect gene required for early embryonic development in the mouse. PLoS One. 2012; 7:e30344.

39. Peng H, Liu H, Liu F, Gao Y, Chen J, Huo J, Han J, Xiao T, Zhang W. NLRP2 and FAF1 deficiency blocks early embryogenesis in the mouse. Reproduction. 2017; 154:145-51. 